\title{
Pathological Worrying and Artificial Neural Networks
}

\author{
Carlos Pelta \\ Dept. of Experimental Psychology, \\ Cognitive Processes and Speech Therapy, UCM Madrid, Spain
}

\begin{abstract}
Worrying is a cognitive process that focuses on potential future negative events, where the outcome is often uncertain. Worries can arise in chains with one worry leading to another, often without solution. This may give rise to an uncontrollable worrying that may be associated with psychiatric disorders such as anxiety and depression. The generation of progressively more negative chains of worries can lead to a catastrophic phenomenon of pathological worrying. In this article we show that catastrophic worrying can be simulated by using a cascade-correlation algorithm for artificial neural networks.
\end{abstract}

Keywords-Pathological worrying; artificial neural networks; cascade-correlation algorithm

\section{INTRODUCTION}

Worrying is a daily experience for most people but this does not mean that it is a simple process. Following a definition by Barlow [1], it is a cognitive process, typically verbal, that focuses on potential future negative events, where the outcome is often uncertain. For Wells and his metacognitive model [2], on the other hand, worrying is connected to metacognitive beliefs (both positive and negative) that imply a recurrent style of thinking linked to people's conscious strategies to deal with threats. Worries are interpreted as cognitions, in the line of cognitive behavior therapy or as metacognitive beliefs [3]. Worries can arise in chains with one worry leading to another, often without solution. This may give rise to an uncontrollable worrying that may be associated with psychiatric disorders such as anxiety and depression [4]. It has been suggested that nonpathological worrying and pathological worrying do not differ in content but in terms of prevalence and severity. Ruscio, Borkovec and Ruscio [5] have examined whether the difference between pathological worrying and normal worrying is one of a kind of degree. According to them, pathological and normal worrying take care of similar topics, but pathological worrying concerns to more issues, is more durable and often is uncontrollable.

The generation of progressively more negative chains of worries can lead to a catastrophic phenomenon [6]. When we think catastrophically, we imagine the imminence of some disaster. Following [7], catastrophism is based on an "overestimation" of the likelihood of a bad result, as well as an "undervaluation" of our ability to deal with the situation correctly. There is a catastrophic level of worrying because stopping the process becomes incompatible with beliefs about the need to worrying and with beliefs about uncontrollability
[8]. A non-adaptive pattern of thought control is developed. This pattern prevents the person from discovering that the worrying process is controllable. Individuals use selfregulation strategies that are counterproductive to reduce worrying.

Using a procedure based on the technique of cognitive therapy of decatastrophizing [9], Vasey and Borkovec [6], found that chronic worriers generated more catastrophic steps than nonworriers and reported a significant increase in discomfort. The worriers spend more time ruminating that nonworriers.

In this study we propose to demonstrate how the use of artificial neural networks can simulate in a very reliable way pathological worry in a sample that mixes worriers and nonworriers subjects. For this, we have used the `Penn State Worry Questionnaire' (PSWQ) in its Spanish version and a catastrophization questionnaire based on the catastrophization interview technique [10]. The results obtained have served to design an artificial neural network that applies a cascadecorrelation algorithm for classifying pathological patients which takes more steps in the catastrophization interview. We show that artificial neural networks in cascade fit perfectly with the process of catastrophization typical of pathological worrying.

The use of artificial neural networks to model neurological and psychiatric disorders is not new. Witnesses to this are classics in the field such as [11] and [12]. They discuss the simulation of neurological disorders ranging from Alzheimer's disease [13] to focal epilepsy [14] and neuropsychiatric disorders ranging from depression [15] to the study of the nature of schizophrenia and autism [16]. In fact, the new field of study of computational psychiatry [17] seems to be a very promising investigation program.

Predictive studies on general anxiety disorder (GAD) are also abundant [18]. However, our study focuses on the prediction of pathological worrying, a type of disorder that is central to the diagnosis of GAD or depression. The psychological disorder of pathological worrying is at the base of much of the psychological suffering and, until now, had not been modeled. Precisely, to this end our study aims to contribute.

We will begin with the selection of the experimental sample through the application of the PSWQ and the catastrophization questionnaire to then propose that what we believe is the best way to model a phenomenon such as the pathological worrying. It is to apply a cascade-correlation algorithm, because the cascading structure of the steps 
conforms to the computational structure of an artificial neural network in cascade. Next we will propose the architecture of our artificial neural network that simulates the phenomenon and we will carry out the evaluation of the training of the network to conclude and in some conclusions and possible applications of the model in the future.

\section{METHOD}

\section{A. Procedure}

We have applied the PSWQ [19] to a sample of $N=86(M$ $=21.6, S D=2.5)$ of high school students night shift $(54 \%$ of female students and $46 \%$ of male students). We have employed its Spanish version [20] which validates the original questionnaire and demonstrates high internal consistency in the case of both clinical and non-clinical criterion groups.

The PSWQ is a questionnaire that assesses worrying as an uncontrollable phenomenon. It is made up of 16 items, which asks participants to rate each item on a 5-point scale ("not at all typical" to "very typical"). Scores range from 16-80 and a higher score represents a greater degree of pathological worry.

Besides we have used an adaptation of the catastrophic interview procedure, focused on the topic "getting pass to the second year course" that gives access to the secondary school diploma. Subjects had to indicate from 0 to 10 their degree of discomfort if they could not pass the course and the probability that it could happen (also on a scale of 0 to 10). Table I shows the catastrophizing sequences generated by a pathological worrier.

\section{B. Results}

The results concerning to the application of the Spanish version of the PSWQ were that $57.1 \%$ of subjects were nonworriers and introduced an average of 8.01 steps in the catastrophization procedure. The average factor of nonworrying was 0.78 . In contrast, $42.9 \%$ showed a pathological worrying index in the PSWQ, validating an average of 14.1 steps and the average factor of worrying was 0.83 . There was a significant difference between the number of steps between the PW (pathological worriers) group and the NW (nonworriers) group $[\mathrm{F}(1,84)=14.075, \mathrm{p}<.05]$. The average degree of discomfort among the pathological worriers was 8.85 on a scale of 10 . On the other hand, the average degree of discomfort among the nonworriers was 6.46. With regard to the average probability of occurrence of the aforementioned events, among the pathological worriers it was 0.81 and among the nonworriers it was 0.58. There were significant differences in degree of worrying between women and men $[\mathrm{F}(1,84)=14.112, \mathrm{p}<.05]$.

\section{ANN Model Design}

In this study we propose to demonstrate how the use of artificial neural networks can predict in a very reliable way the distinction between pathological worriers and nonworriers in a sample of students. We have designed an artificial neural network that applies a cascade-correlation algorithm that fits very well with the process of catastrophization, typical of pathological worriers. The cascade-correlation architecture [21] adds hidden neurons one by one in the network. In the process of adding hidden neurons to the network, each new neuron receives a synaptic connection from each of the input neurons and also from the hidden neurons that precede it. After adding each new hidden neuron, the synaptic weights of its inputs are frozen, while the weights of its outputs are repeatedly trained. This process continues until a desired performance is achieved [22]. It is evident that the cascade architecture allows adding each hidden neuron at a time and only the new weights are updated; the incremental or constructive learning allows to create the new hidden units, where for each new hidden neuron, the algorithm maximizes the magnitude of the correlation between the new hidden neuron and the residual network error, that is, hidden neurons are added trying reduce the network error until its performance is satisfactory.

We have created a cascade backpropagation artificial neural network that presents two input neurons (one that encodes nonworriers-NWI- and the other encodes pathological worriers-PWI-), a layer hidden (H1) of 8 neurons (C1-C8) and 7 hidden (H2-H8) cascading neurons (CPW1-CPW7) that are added to the successive hidden layers culminating in an output that encodes the pathological worriers' output (PWO). The output that encodes nonworriers (NWO) is connected to the 8 cascading neurons (C1-C8) of the first hidden layer (H1). Actually, with the seven hidden $(\mathrm{H} 2-\mathrm{H} 8)$ cascading neurons (CPW1-CPW7), we have wanted to reflect the steps of more than, in the questionnaire, pathological worries had added. As shown in Fig. 1, our network has a pool of 15 candidates forming the layers of hidden neurons, two inputs (one exemplifying the pathological worries and the other exemplifying the nonworriers) and two outputs (to reflect the results of the pathological worrying and non worrying, respectively).

A sample of one pattern visualization showing the activation of PWI, PWO and of the hidden neurons is presented in Fig. 2.

TABLE. I. CATASTRophic SEQUENCES

\begin{tabular}{|l|l|l|l|}
\hline \multirow{2}{*}{ Steps } & \multicolumn{3}{|l|}{ If I don't get pass to the second year course } \\
\cline { 2 - 4 } & Catastrophizing sequence & Discomfort & Likelihood \\
\hline 1 & I won't live up to my expectations. & 9 & 9 \\
\hline 2 & $\begin{array}{l}\text { It would be harder to study what I } \\
\text { don't like. }\end{array}$ & 10 & 9 \\
\hline 3 & $\begin{array}{l}\text { It would be harder to get a good } \\
\text { job for me. }\end{array}$ & 10 & 8 \\
\hline 4 & My salary would be lower. & 9 & 9 \\
\hline 5 & It would demotivate me a lot. & 10 & 9 \\
\hline 6 & I'd lose my self-confidence. & 8 & 8 \\
\hline 7 & $\begin{array}{l}\text { My loss of confidence would } \\
\text { affect other areas of my life. }\end{array}$ & 9 & 8 \\
\hline 8 & I'd become very anxious. & 9 & 9 \\
\hline 9 & $\begin{array}{l}\text { I'd feel like I wouldn't have any } \\
\text { control over my life. }\end{array}$ & 10 & 8 \\
\hline 10 & I'd feel mentally bad. & 10 & 8 \\
\hline 11 & I'd be very unhappy. & 10 & 7 \\
\hline 12 & I'd like to die. & 10 & 6 \\
\hline
\end{tabular}




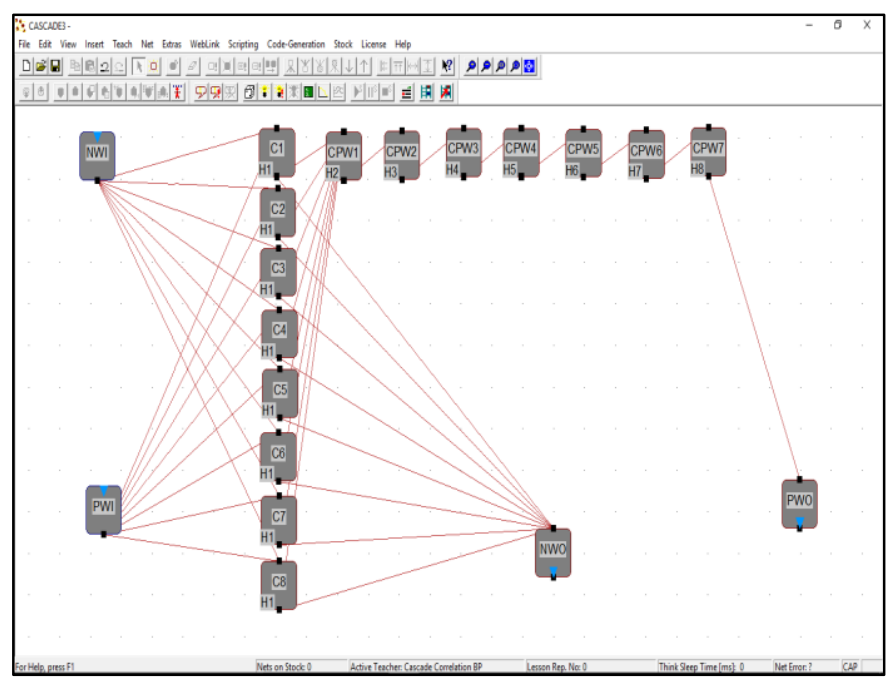

Fig. 1. The Proposed ANN Architecture.

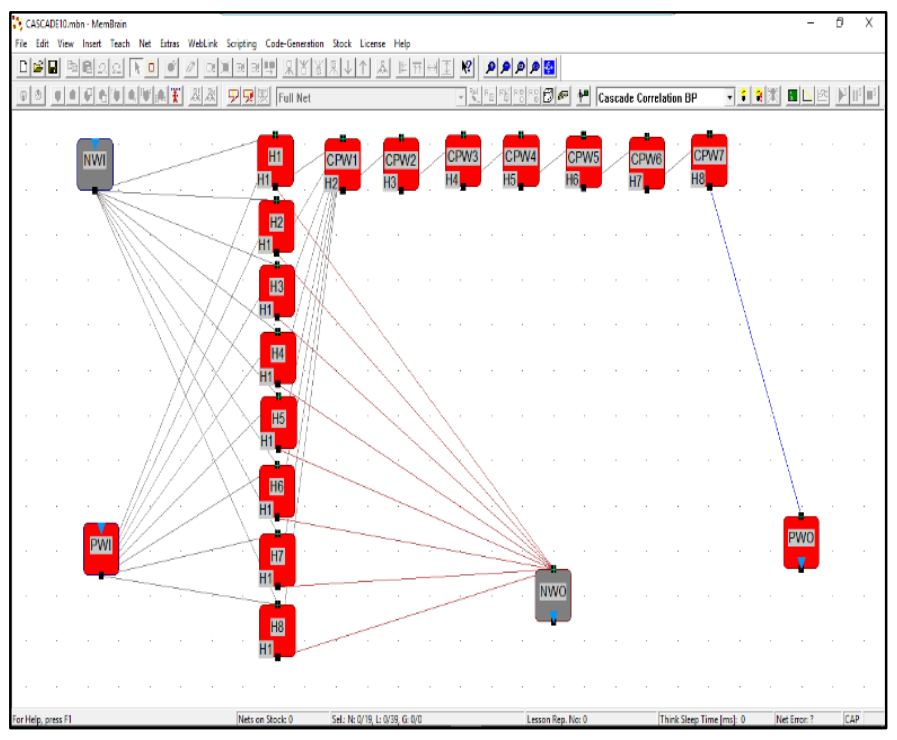

Fig. 2. Sample of Pattern Visualization.

\section{ANN EVALUATION RESUlts}

MemBrain (version V03.08.01.00), a framework designed to develop artificial neural networks was used in training our ANN model. It contains (see [23], [24], [25]) an implementation for most of the learning algorithms including cascade-correlation using backpropagation. We have used a Sigmoid (logistic) function as transfer function with values between -0.5 and 0.5 . As observed in Fig. 3, learning rate was 0.25 and target net error was 0.01 .

The learning rate parameter determines the size of the weights adjustment each time the weights are updated during training [26].

The initial weights have been matched to the probabilities with which the subjects have token the successive steps in the catastrophization questionnaire (see Table II).

Error measure has been then computed to assess the neural network's accuracy. Mean Squared Error (MSE) has been used yielding the smallest value of MSE equal to 0.00986309276170759 for nonworriers and the smallest value of MSE equal to 0.00974693104479933 for pathological worriers (see Table III).

Fig. 4 and 5 show the Net Error Graph for nonworriers and pathological worriers.

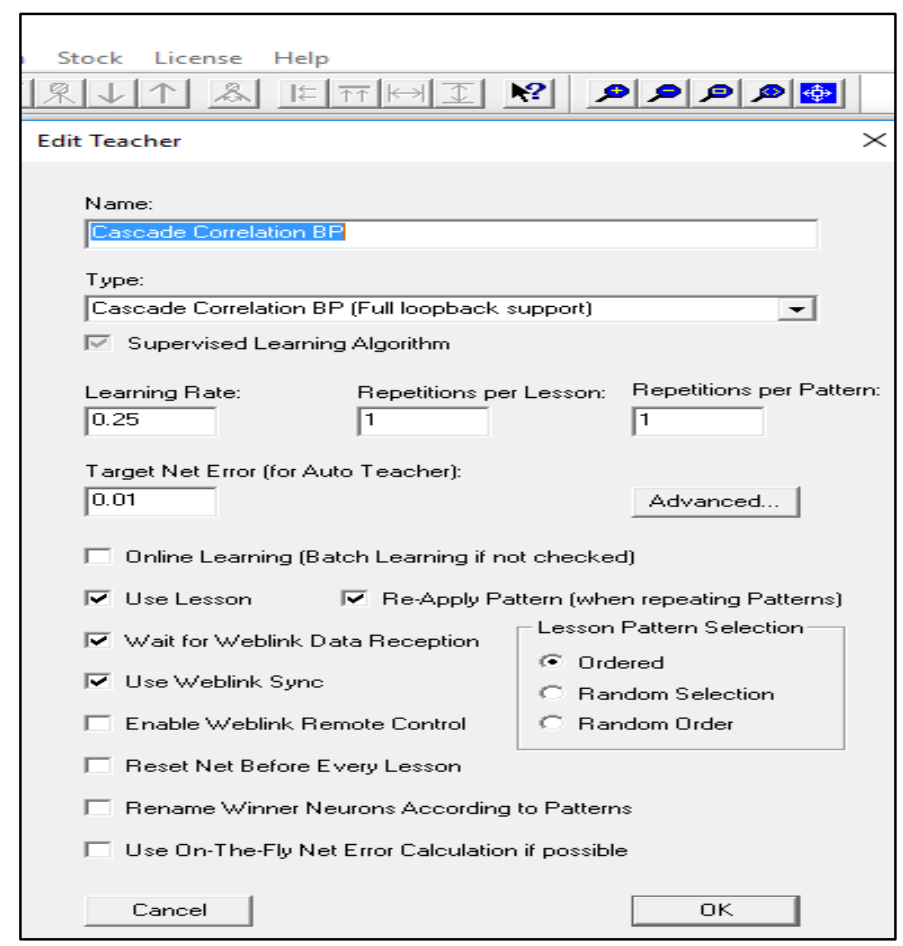

Fig. 3. View of "Edit Teacher" Tool.

TABLE. II. InITIAL WeIghts C1 C2 C3 C4 C5 C6 C7 C8 CPW1 CPW2 CPW3 CPW4 CPW5 CPW6 CPW7 C CANDIDATE NeURONS

\begin{tabular}{|c|c|c|c|c|c|c|c|c|c|c|c|c|c|c|c|c|}
\hline Input layers & PWI & 0.57 & 0.60 & 0.68 & 0.71 & 0.75 & 0.78 & 0.82 & 0.89 & 0.83 & 0.87 & 0.94 & 0.98 & 0.72 & 0.75 & 0.65 \\
\hline Input layers & NWI & 0.13 & 0.15 & 0.22 & 0.23 & 0.27 & 0.28 & 0.34 & 0.37 & & & & & & & \\
\hline Output layers & PWO & & & & & & & & & 0.84 & 0.89 & 0.95 & 0.99 & 0.74 & 0.76 & 0.71 \\
\hline Output layers & NWO & 0.15 & 0.16 & 0.24 & 0.27 & 0.31 & 0.34 & 0.36 & 0.41 & & & & & & & \\
\hline
\end{tabular}


TABLE. III. MEAN SQUARED ERRor

\begin{tabular}{|l|l|l|}
\hline $\boldsymbol{A N N}$ & Pathology & $\boldsymbol{M S E}$ \\
\hline Cascade & Nonworriers & 0.00986309276170759 \\
\hline Cascade & Pathological worriers & 0.00974693104479933 \\
\hline
\end{tabular}

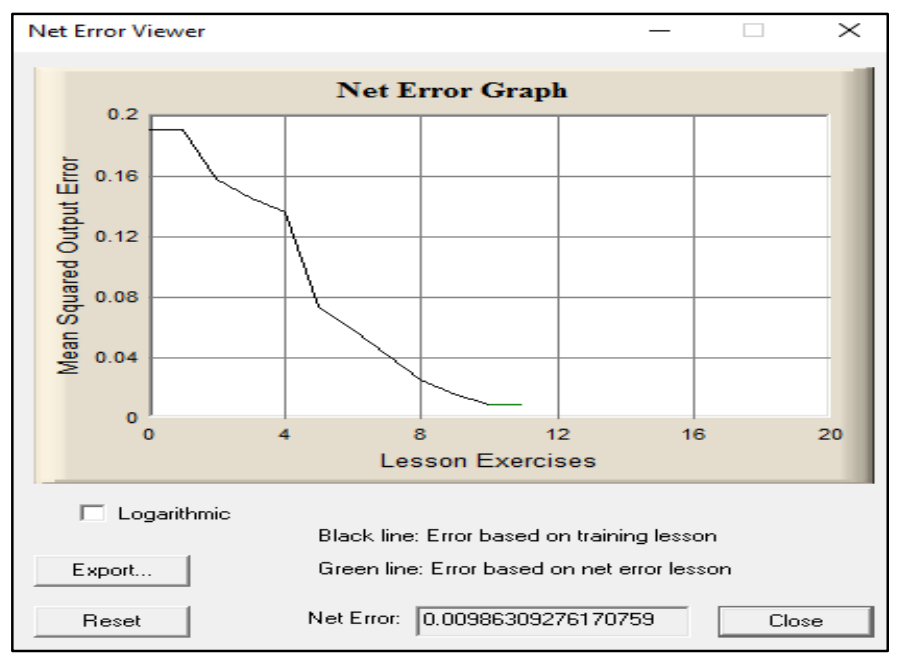

Fig. 4. Net Error Graph for Nonworriers.

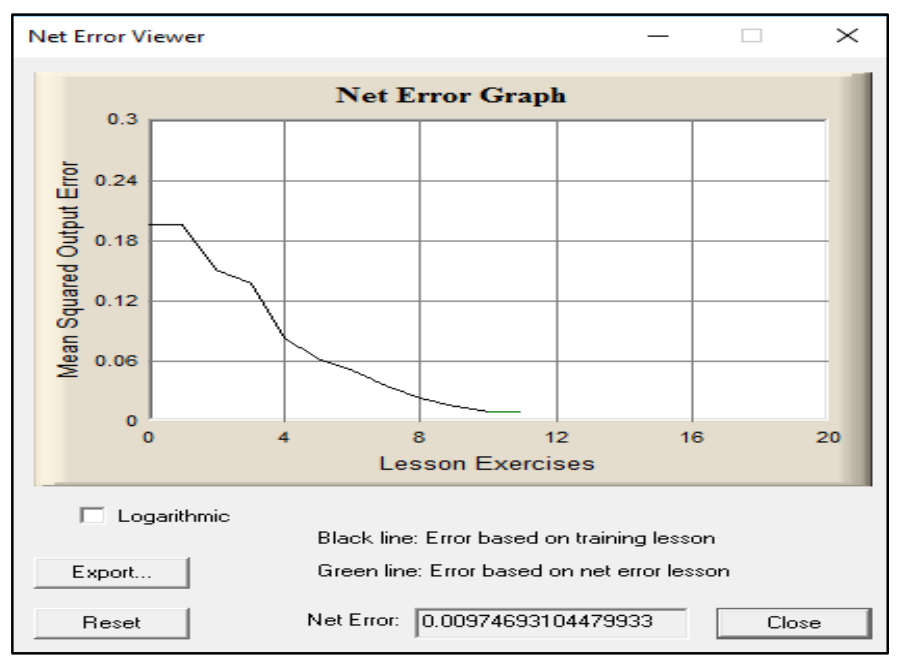

Fig. 5. Net Error Graph for Pathological Worriers.

\section{CONCLUSIONS}

This study has attempted to build a suitable ANN model for modeling the phenomenon of worrying distinguishing between nonworriers and pathological worriers. The type of artificial neural network used has been a cascade-correlation network with backpropagation. The backpropagation algorithm modifies network weights to minimize the mean squared error between the desired and the actual outputs of the network. But cascade-correlation enables to create a constructivist topology [27] of hidden layers that reflects the catastrophization process typical of pathological disorders like worrying, rumination or chronic anxiety. Cascade-correlation seems more in tune with the simulation of events involving intense emotional cycles [28]. These cycles are selfamplifying positive feedback loops of strong negative emotions. Cascading networks grow in a similar manner to the process of catastrophization in psychiatric disorders and mimic its sequential growing in steps.

There were some limitations to note. First, a relatively small sample size and low reports of some behaviors. Further, measures of worrying used here need additional validation. It would be necessary to determine if worrying is actually difficult to control and to examine specific aspects of negative emotion and rumination that may be involved in pathological worrying.

A future task should be to simulate, by using cascadecorrelation artificial neural networks, mental pathologies such as pathological rumination [29] or borderline personality disorder [30].

\section{REFERENCES}

[1] D. Barlow, Anxiety and its disorders: The nature and treatment of anxiety and panic, 2nd ed. New York: Guilford Press, 2004.

[2] A. Wells, "The metacognitive model of worry and generalised anxiety disorder," in Worry and its psychological disorders: Theory, assessment and treatment, G. T. L. Davey and A. Wells (Eds.) Chichester: John Wiley\&Sons, 2006, pp.179-199.

[3] A. Beck, Depression: clinical, experimental and theoretical aspects. New York: Harper and Row, 1967.

[4] M.G. Newman, S.J. Llera, T.M. Erickson, A. Przeworski, and L. G. Castonguay, "Worry and generalized anxiety disorder: a review and theoretical synthesis of evidence on nature, etiology, mechanisms, and treatment,” Annu. Rev. Clin. Psychol., vol. 9, pp. 275-297, March 2013. https://doi.org/: 10.1146/annurev-clinpsy-050212-185544.

[5] A.M. Ruscio, T.D. Borkovec,, and J. Ruscio, "A taxometric investigation of the latent structure of worry. Abnormal Psychology, 110(3), pp. 413-422, 2001. http:// dx.doi.org/10.1037//0021843X.110.3.413.

[6] M.W. Vasey and T.D. Borkovec, "A catastrophizing assessment of worrisome thoughts". Cogn. Ther. Res., 16(5), pp. 505-520, 1992. https://doi.org/10.1007/BF01175138.

[7] E. Bourne and L. Garano, Coping with anxiety: ten simple ways to relieve anxiety, fear, and worry, 2nd ed., Oakland: New Harbinger Publications, 2016.

[8] A. Wells, "Metacognition and worry: a cognitive model of generalised anxiety disorder", Behavioural and Cognitive Psychotherapy, 23, pp. 301-320, 1995.

[9] P.C. Kendall and R.E. Ingram, "The future for cognitive assessment of anxiety: let's get specific," in Anxiety and stress disorders: Cognitivebehavioral assessment and treatment, L. Michaelson and L.M. Ascher (Eds.) New York: Guilford Press, 1987, pp. 89-104.

[10] G.C.L. Davey, "The catastrophising interview procedure," in Worry and its psychological disorders: Theory, assessment and treatment, G. T. L. Davey and A. Wells (Eds.) Chichester: John Wiley\&Sons, 2006, pp.157-176.

[11] J.A. Reggia, E. Ruppin and R. Sloan Berndt (Eds.), Neural modeling of brain and cognitive disorders. Singapore: World Scientific, 1996.

[12] D.J. Stein and J. Ludik (Eds.), Neural networks and psychopathology. Cambridge: CUP, 1998.

[13] E. Ruppin, D. Horn, N. Levy, and J.A. Reggia, "Computational studies of synaptic alterations in Alzheimer's disease", in Neural modeling of brain and cognitive disorders, J.A Reggia, E. Ruppin and R. Sloan Berndt (Eds.) Singapore: World Scientific, 1996, pp. 43-62.

[14] M.R. Mehta, C. Dasgupta and G.R. Ullal, "A neural network model for kindling of focal epilepsy", in Neural modeling of brain and cognitive disorders, J.A. Reggia, E. Ruppin and R. Sloan Berndt (Eds.) Singapore: World Scientific, 1996, pp. 347-366.

[15] M. Raeiati Banadkooki, C. Mielke, K. H. Wolf, R. Haus and M. Marschollek, "Automatic detection of depression by using a neural network", Stud. Health Technol. Inform., 251, pp. 3-6, 2018. 
[16] P. Lanillos, D. Oliva, A. Philippsen, Y. Yamashita, Y. Nagai and G. Cheng "A review on neural network models of schizophrenia and autism spectrum disorder", Neural Networks, 122, pp. 338-363, 2020. https://www.sciencedirect.com/science/article/pii/S0893608019303363T

[17] A.D. Redish and J. A. Gordon (Eds.), Computational psychiatry: new perspectives on mental illness. Cambridge: Mass.: The MIT Press, 2016.

[18] M. Sribala, "An approach of artificial neural networks for prediction of generalized anxiety disorder", IJRCAR, 3(3), pp.118-124, 2015.

[19] T.J. Meyer, M.L. Miller, R.L. Metzger and T.D. Borkovec, "Development and validation of the Penn State Worry Questionnaire," Behaviour Research \& Therapy, 28, pp. 487-495, 1990.

[20] B. Sandín, P. Chorot, R.M. Valiente and L. Lostao, "Validación española del cuestionario de preocupación PSWQ: estructura factorial y propiedades psicométricas," Revista de Psicopatología y Psicología Clínica, vol. 14(2), pp. 107-122, 2009.

[21] S.E. Fahlman and C. Lebiere, "The cascade-correlation learning architecture," in Advances in neural information processing systems 2, D.S. Touretzky, Ed., Los Altos, Calif.: Morgan Kaufmann, 1990, pp.524-532.

[22] L. Pretzchel, "Early stopping-but when?, in Neural networks: tricks of the trade, G. Montavon, G. Orr and K.-R. Müller (Eds.) Berlin, Springer, 2012, pp.53-67.
[23] MEMBRAIN - NN.DE. https://membrain-nn.de/main_en.htm.

[24] A. Popko, M. Jakubowskl and R. Wawer, "MemBrain neural network for visual pattern recognition," ASTRJ, 7(18), June 2013, pp. 54-59. https:// doi-org/:10.5604/20804075.10512571990.

[25] A. Popko, "Optical horizon recognition using artificial neural networks," ICCSEAS, November 2017, pp. 33-38, 2017.

[26] S. Haykin, Neural networks: A comprehensive foundation, 2nd ed. Upper Saddle River, N.J.: Prentice-Hall, 1999.

[27] T.R. Shultz, Computational developmental psychology. Cambridge, Mass.: The MIT Press, 2003.

[28] E.A. Selby, M.D. Anestis and T.E. Joiner, Jr., "Understanding the relationship between emotional and behavioral dysregulation: emotional cascades," Behaviour Research \& Therapy, 46, pp. 593-611, 2008. https.//doi.org/: 10.1016/j.brat.2008.02.002.

[29] C. Papageorgiou and A. Wells, Eds., Depressive rumination: Nature, theory and treatment. Chichester: John Wiley\&Sons, 2004.

[30] E.A. Selby and T.E. Joiner, Jr., "Emotional cascades as prospective predictors of dysregulated behaviors in borderline personality disorder," Personality Disorders: Theory, Research and Treatment, 4(2), pp. 168174, 2013. https://doi.org/10.1037/a0029933. 